Indexaciones: Repositorio de Revistas UCR, DIALNET, Latindex, REDALYC Directorio y recolector de recursos digitales del Ministerio de Cultura de España, Directory of Open Access Journals. Diálogos Revista Electrónica de Historia ISSN 1409-469X. Número especial 2008. Dirección web: http://historia.fcs.ucr.ac.cr/dialogos.htm

\title{
Tierra Blanca una montaña de esperanza en la cura de la tuberculosis
}

\author{
Carmela Velásquez Bonilla
}

Doctora en Historia por la Universidad de Costa Rica.

Docente de la Escuela de Historia e investigadora del

Centro de Investigaciones Históricas de América Central, ambos de la misma universidad. Correo electrónico: carmelav@racsa.co.cr 


\section{Introducción}

A fines del siglo XIX y durante las primeras décadas del siglo XX, en Costa Rica fue evidente la búsqueda de un mejoramiento de las condiciones higiénicas de la población. Como señala Steven Palmer, una generación de médicos profesionales costarricenses, que surgió en las últimas décadas del siglo XIX. Tuvo un papel protagónico y fundamental en la aplicación de políticas de salud pública y en la modernización del aparato del Estado costarricense . 1

Entre los logros obtenidos se señalan la modernización del Hospital San Juan de Dios, la creación del Asilo Chapuí, la fundación de un sanatorio para tuberculosos, que llamaron Sanatorio Carit y la Escuela de Enfermería. Por iniciativa de este grupo de profesionales no solo se crearon los centros, sino que se buscó el financiamiento para construirlos y asegurar su mantenimiento. Fue así como surgió la Lotería Nacional con el fin de conseguir ingresos y La Junta de Caridad para velar por los fondos de aquellos centros de enseñanza y de salud.

Por eso, se ha considerado relevante que a partir de la investigación sobre uno de los centros de salud mencionados, el sanatorio de tuberculosos Carit en Tierra Blanca de Cartago, se pueda establecer el grado de avance de la medicina en Costa Rica en comparación con los parámetros mundiales de la época.

Esa situación fue posible sobre todo por el interés de los médicos en continuar estudiando, de mantenerse al día con la lectura de libros y de las últimas revistas que venían del exterior como “The Lancet”, revista inglesa, que comentaban en sus sesiones de estudio en el Hospital San Juan de Dios. ${ }^{2}$ Además, se traducían los artículos científicos para ser publicados en La Gaceta Médica.

Comparando la situación de la medicina costarricense con el resto de Centro América, se puede establecer, por ejemplo, que a nivel del tratamiento de la tuberculosis, no existía en la región un sanatorio como el Carit. Al respecto en Congresos Médicos de Centro América efectuados antes de 1938, fecha de la fuente, se señaló lo siguiente:

"Un distinguido médico salvadoreño, desaparecido ya, patrocinó en un tiempo la idea de un sanatorio para tuberculosos en "Los Altos” de Guatemala costeado por esta república

y El Salvador. Todas estas son manifestaciones de un verdadero centro-americanismo,

1 Steven Palmer.From Popular medicine to medical populism. Doctors, healers and public power in Costa Rica, 1800-1940. (Durham and London: Duke University Press, 2003) 67. Citado en Quesada Florencia, La modernización entre cafetales. (San José Costa Rica, 1880-1930), 105-106.

2 Carmela Velázquez, Diálogos, Revista Electrónica de Historia ISSN 1409-469X Vol7) Número 7

Febrero 2006-Agosto 2006 
nacido de la comunidad de ideales y fortalecido ante las necesidades que sienten las repúblicas hermanas al enfrentarse ante los problemas que su bienestar social implica. Una acción conjunta y una cooperación parecidas podrían invocarse en favor de la educación médica en Centro América.”3

Esto muestra que era necesario un sanatorio para tuberculosos en la región centroamericana y que la propuesta de construirlo en conjunto en la zona de "Los Altos" que es un terreno a buena altura sobre el nivel del mar, manifiesta el conocimiento que se tenía sobre otros sanatorios del mundo en ese momento. En el caso de Nicaragua, la propuesta de un sanatorio para el tratamiento de la tuberculosis fue aprobada hasta 1956 a solicitud de la Junta Nacional de Asistencia Social, cuando era presidente de la república Anastasio Somoza García. Por lo que el Poder Ejecutivo de esa nación accedió a lo siguiente:

"la creación de un moderno Sanatorio para Tuberculosos cuyo número en el país ha ido en aumento durante el curso de los últimos años. Por Cuanto: No existe en la actualidad ningún establecimiento de esa clase destinado a la atención de las enfermedades pulmonares, que reúna todas las condiciones exigidas por la ciencia médica y que esté servido por medio de las Instituciones de Asistencia Social; De los estudios tanto médicos, como de ingeniería, que se han hecho, el lugar que reúne las mayores facilidades para una obra de esa naturaleza ese lugar llamado Saratoga en la jurisdicción de Catarina, del Departamento de Masaya." ${ }^{4}$

Esta información muestra lo que tardó en establecerse un sanatorio en Nicaragua y, como muy bien lo indica el documento citado, no había un antecedente al respecto. Es conveniente señalar que la zona propuesta para este sanatorio se encuentra a una altura conveniente y que es un lugar de fuertes vientos, lo que estaba dentro de las propuestas mundiales para la creación de centros para el cuidado de los tuberculosos.

3 Revista hondureña Año VIII N 78 de 1938. Consulta 25 de abril del 2008.http://cidbimena.desastres.hn/ RMH75/pdf/1938/pdf/A8-5-1938-1.pdf.

4 legislación.asamblea.gob.ni/Normweb.nsf/ 0/575730a7cf8f0bd70625718c004aa4a7.consulta 25 de abril del 2008. 
En el caso de Panamá, por medio de la Ley 133 de 1943 se ordenó la construcción de un hospital para tuberculosos.

Otro aspecto interesante de mencionar es que en España solo existían seis sanatorios, cuando Costa Rica construía el suyo.

Lo anterior permite confirmar que Costa Rica tenía en ese momento (finales s. XIX y principios s. XX) una medicina muy adelantada y que quienes la practicaban, los médicos, tuvieron la gran visión de llevar a la realidad la puesta en práctica en centros especializados como el Sanatorio Carit, el Asilo Chapuí y el Hospital San Juan de Dios, los avances por ellos aprendidos, la mayoría en el exterior.

\section{La tuberculosis}

La tuberculosis es una enfermedad pulmonar conocida en los primeros años de la Edad Moderna como "tisis” o "consunción” dado que parecía que consumía a sus víctimas y dejaba esqueletos a su paso. El bacilo responsable de esta enfermedad era el Mycrobacterium tuberculosis descubierto por el alemán Koch en 1882. De transmisión aerógena, provoca la reacción inflamatoria de los tejidos orgánicos infectados. El tubérculo constituye su lesión elemental y los pulmones, su principal localización, seguida del intestino, los ganglios linfáticos, la piel, los órganos genitales-urinarios y los huesos. ${ }^{5}$

Hasta el siglo XVII, el conocimiento sobre la tuberculosis apenas cambió. Los médicos árabes como Razes y Avicena la consideraban como una afección generalizada con manifestaciones locales en forma de úlceras pulmonares. Arnau de Vilanova pensaba que se originaban en humores fríos que caían gota a gota desde la cabeza a los pulmones. Su incidencia en esta época es difícil de concretar debido a la ausencia de estadísticas. ${ }^{6}$

En la Edad Media, no hay que dejar de lado la concepción de que las enfermedades eran identificadas con el pecado, con el mal y con los demonios y percibidas como un castigo, por lo que la ayuda celestial era muy importante. ${ }^{7}$ No se ha podido determinar un santo específico al que se acudía para la sanación de la tuberculosis como el caso de San Blas para la garganta, pero sí se señala que muchos enfermos visitaron el Santuario de Lourdes, Francia, en búsqueda 5 José Luis Beltrán Moya, Historia de las epidemias en España y sus colonias (1348-1919).( España: Cofas, 2006), 157.

$6 \quad$ Ibid., 157.

$7 \quad$ Carmela Velázquez, El sentimiento religioso y sus prácticas en la Diócesis de Nicaragua y Costa Rica. (Tesis de doctorado del sistema de estudios de posgrado de la Universidad de Costa Rica, 2004), 328-329. 
de la cura. En Francia y en Inglaterra en el medioevo, se celebraba el ceremonial de los reyes taumaturgos, que no era otra cosa que la curación de la escrófulas que eran tocadas por las manos de los reyes quienes repetían la frase: "El Rey te toca, Dios te cura. ${ }^{8}$ De esa manera se pedía la intervención divina para sanarse.

La ritualidad que rodeaba la curación de los enfermos de tuberculosis practicada por los reyes taumaturgos ${ }^{9}$ nació en el año 1000 y un año después empezó a practicarse en Inglaterra. Al viejo gesto mágico de bendecir se le agregó otro también tradicional de su tiempo, pero específicamente cristiano, la señal de la cruz sobre los pacientes o sobre las heridas. ${ }^{10}$ Explica Bloch que con el tiempo el rito se fue volviendo cada vez más rico y, en el caso de los ingleses, la ceremonia se convirtió casi en un servicio litúrgico y el rey parecía un oficiante; además, el rey repartía sus bendiciones.

Con respecto a la ritualidad que rodeaba la curación, es pertinente señalar que el rey utilizaba los días que eran las principales fechas del año religioso, por ejemplo, la Candelaria, el Domingo de Ramos, la Pascua o uno de los días de Semana Santa, Pentecostés, la Ascensión, Corpus Christi, la Asunción, la Natividad de la Virgen, Navidad; por excepción tuvo lugar en días que no eran del calendario litúrgico. El rey además tenía la costumbre de comulgar bajo las dos especies utilizando sus manos, según este privilegio dinástico, antes de la ceremonia de imposición de manos y con el don de curar, parecía afirmar el carácter sagrado de la monarquía francesa. ${ }^{11}$

Ya en el siglo XVII médicos como Silvio, el gran clínico holandés, quien por medio de la autopsia estableció una relación entre la tisis y su alcance pulmonar; también, Thomas Willis y Richard Morton dieron su aporte a esta investigación. El primer paso en el diagnóstico fue dado por Avenbrugger en 1761 mediante el uso de la percusión torácica, técnica que más tarde, en 1797, fue difundida por Jean Nicole Corvisart, médico de Napoleón Bonaparte. Posteriormente, los estudios de Gaspart Laurent Bayle en 1810 y Hermann Lebrut en 1849 antecedieron al descubrimiento de Koch en 1882 del microorganismo causante de la tuberculosis. ${ }^{12}$

$8 \quad$ Marc Bloch. Los reyes taumaturgos (México, Fondo de Cultura Económica,1988), 94.

9 Taumaturgo es aquel que cura por medio del contacto, en este caso por medio de las manos. Cuando se refiere a imagines son las que las personas les guardan una especial fe y por eso las tocan esperanzados en que su favor les llegue por medio del contacto y pore so llaman a esta imágenes taumaturgas.

$10 \quad$ Ibid.,80.

11 Citado en Carmela Velásquez, El sentimiento religiosos y sus practices en la Diócesis de Nicaragua y

Costa Rica. Siglos XVII y XVIII, 14

12 Jose Luis Beltran,157. 
La tuberculosis tuvo su época más fuerte entre 1780 y 1880, la enfermedad incidió sobre todo en adultos jóvenes, quienes la mitificaron y la convirtieron en una enfermedad de moda. La palidez, el brillo febril de la mirada y la astenia que acompañaban a la tisis la convirtieron en la enfermedad de las almas sensibles y de los artistas: Chautobriand, Chopin, Geoges Sand, Alfred de Musset, Gaspar D. Friedrich y Gustavo Adolfo Bécquer, fueron algunos de los exponentes de esa filosofía estética de la enfermedad. ${ }^{13}$ También sufrieron la enfermedad grandes políticos como Simón Bolívar y José Nariño, al igual que los santos San Francisco de Asís y San Luis Gonzaga entre otros.

También algunos casos de esta enfermedad fueron inspiradores de grandes obras de la literatura, del teatro y hasta de una ópera. Thomas Mann a raíz de una visita a su esposa que estaba internada en un sanatorio para tuberculosos en los Alpes suizos, empezó a escribir en 1912, La montaña mágica, que se conoce como su novela más importante y un clásico de la lengua alemana del siglo XX, que ha sido traducida a numerosos idiomas. ${ }^{14}$ Su argumento tiene como escenario el Sanatorio Internacional Berghof en los Alpes suizos a más de 1.600 metros de altura. El eje lo constituye un joven que llega a internarse en el sanatorio, y relata toda la vida cotidiana que se desarrolla en ese mundo cerrado que es un sanatorio, cerrado, porque ahí solo llegan los enfermos y los visitantes, pero muy abierto al aire, al que se le atribuían grandes beneficios de curación.

Otra gran obra fue la novela de Víctor Hugo hijo, La dama de las camelias publicada en 1848, basada en la vida de una cortesana de París que se llamaba Marie Duplessis, mujer de una gran belleza por la que fue asediada por gran cantidad de pretendientes. Tuvo un romance con Víctor Hugo hijo, luego se separaron y ella volvió después de un matrimonio fallido a su vida en París y murió de tuberculosis a los 23 años. En la dama de las camelias, el personaje femenino fue la cortesana Marguerite Gautier, que tuvo un amante Armand Duval que para tratar de curarla de la peste blanca la llevó al campo, pero el padre de Armand le pidió a Marguerite que lo dejara por el bien del muchacho. Ella para cumplir su promesa al padre de Armand, lo dejó y volvió a la vida agitada de París y ahí murió tuberculosa. Con este argumento se han realizados películas y obras de teatro. Además está obra fue la base del argumento del gran compositor italiano Giusepe Verdi para crear la excelente ópera, La Traviata.

$13 \quad$ Ibid.,158

14 es.wikipedia.org/wiki/Thomas_Mann_56K Consulta 28 de abril del 2008 


\section{La búsqueda de la cura}

La enfermedad atacó a todas las clases sociales, las familias que tenían enfermos los escondían, el enfermo pasó a ser un marginado social, su tos, su sudor, todo lo que tocaba era observado como un peligro de difusión del contagio, por lo que, se desinfectaban sus casas y se quemaban sus pertenencias. Si bien se pensaba que la cura estaba basada en el clima puro y el sol, fue hasta 1854 que se empezaron a crear los sanatorios, el primero se hizo en Gorbersdof (Silesia), en los Alpes germanos, a 650 metros de altitud. El doctor Hermann Brehmer sostenía que la tisis era el resultado de la incapacidad del corazón de hacer circular la sangre y así se pasaba a ser tuberculoso por esa razón, el enfermo mejoraría con la reducción de la presión atmosférica que incrementaría la función cardiaca y, con ello la circulación pulmonar. El funcionamiento del corazón lo complementaría una dieta rica y abundante, algo de alcohol, hidroterapia y ejercicio físico regular, todo bajo vigilancia médica constante. Fue así que tanto Chopin como Bécquer estuvieron en periodos de cura en las montañas. ${ }^{15}$

Eso llevó a pensar que para curar a los tísicos lo más importante no era solo la altura, sino condiciones climáticas más adecuadas. En consecuencia, el aspecto fundamental del tratamiento debía aprovechar en cada país los lugares y climas apropiados para construir residencias en las que los pacientes pudieran aislarse durante varios meses experimentando la acción curativa del aire puro, el reposo absoluto y la alimentación abundante y variada. ${ }^{16}$

Bajo esta teoría se empezaron a crear sanatorios en las diferentes montañas del mundo, en Francia en Le Reved (1200 m), Chamonix (1050 m) y Torrench (1250 m); en Suiza: en Davos (1559 m), en Saint Luc (1643) y en Montana (1520 m). En los Estados Unidos de Norte América según una comunicación de "The Nacional Asociation for the Study and Prevention of Tuberculosis" dirigida al Sanatorio Carit consideraba que era necesario una altura de 4.000 pies (medida inglesa), es decir, 1219,17 m. ${ }^{17}$ En España, el primer sanatorio se abrió en 1897 en Busot, Alicante y ya para 1918 existían seis, tres en Madrid, uno de ellos privado y el resto en Barcelona, Valencia y Zaragoza.

$15 \quad$ Betran.159

16 Vivian Solano. La tuberculosis y la cura sanatorial en Costa Rica:el caso del sanatorio Carlos Durán 1915-1940. Sistema de Estudios de posgrado de maestría professional de la Universidad de Costa Rica. 2004, 32. 17 Sin Autor, Sanatorio Nacional de tuberculosos en Cartago, República de Costa Rica, América Central. Sanatorio Carit.(San José, 30 de junio del 2008) 27 


\section{La creación del Sanatorio Carit en Tierra Blanca de Cartago}

La tuberculosis era una enfermedad endémica en Costa Rica que había contribuido eficazmente al alto índice de mortalidad del país. Su tratamiento adecuado era prácticamente nulo al no contarse con instalaciones, equipo y personal idóneo que permitieran asistir al enfermo como lo recomendaba su enfermedad. ${ }^{18} \mathrm{Al}$ respecto el doctor Durán mostró su interés por la creación de un sanatorio desde 1904 cuando como munícipe su colega y amigo el doctor Luis Paulino Jiménez le propuso la construcción de un centro para tratar la enfermedad. ${ }^{19}$ La idea no tuvo frutos en ese momento, pero el doctor no dejó de pensar en ella y siguió insistiendo hasta realizarla.

Además, en la creación del Sanatorio Carit, influyó también una circunstancia personal del doctor Carlos Durán, su hija Elena, la tercera de sus seis hijos, sufrió el ataque de la tuberculosis. Ante esta situación, Durán decidió investigar las diferentes opciones para escoger el mejor centro de curación para llevar a su hija, por lo que acudió a sus fuentes médicas para informarse.

El doctor Durán no se contentó con el conocimiento que había obtenido en la aulas universitarias, ${ }^{20}$ pues no solo se preocupó por la biblioteca de la Academia, sino que en su casa se encontraban revistas de las más recientes, en aquel entonces, sobre medicina y cirugía. Estas revistas eran leídas y analizadas por él y al margen de ellas escribía a lápiz sus comentarios. Se decía que por sus vastos conocimientos era uno de los más aventajados médicos del protomedicato. Tuvo también por costumbre visitar, en diferentes épocas de su vida clínicas y hospitales de Canadá, Estados Unidos y Europa para conocer los últimos avances médicos.

El espíritu de renovación del doctor Durán lo destacó el doctor Ricardo Moreno Cañas en la ceremonia realizada, en 1925 para develar el retrato del doctor Durán en el salón de actos de la Facultad de Medicina de Costa Rica. En esa ocasión, señaló que para él y para los médicos, que habían llegado recientemente de exterior después de efectuar sus estudios, era motivo de admiración observar que este médico de cabellos blancos, pero de mirada y pulso firmes, no solo era un trabajador sin rival, sino que el mejor enterado de los progresos de la medicina.

Esta tenacidad de estudio le permitió decidirse por el sanatorio Loomis en Liberty en New York, para llevar a Elena. El sanatorio había sido creado y dirigido por el doctor Charles Loomis,

18 Germán Solís Barquero. El doctor Durán C. Su participación en la política costarricense.( Tesis de licenciatura de la Universidad de Costa Rica, 1974),163.

19 Ibid.,163.

20 Velázquez, Diálogos VII...114 
la máxima autoridad en tuberculosis en los Estados Unidos en aquel momento; había estudiado en Inglaterra en el Guys Hospital como Durán. La dedicación de Loomis para curar la tuberculosis fue motivada por la necesidad de curarse, ya que él sufrió la “tisis” y logró sobrevivirla.

Durán partió para New York el 19 de mayo de 1903, en barco alemán Altoi con su esposa y cuatro de sus hijos, el barco viajaba vía Kingston al puerto de New York. ${ }^{21}$ Es muy posible que de ahí tomara el ferrocarril hacia Liberty a 105 millas al norte de New York. Se especula sobre la posibilidad de que tomara el ferrocarril, ya que en esa época era el medio usual de transporte y además entre las postales que trajo Durán de su viaje se encuentra la estación del tren en Liberty.

Es muy probable que el doctor Durán tan inquieto por los avances de la medicina se dedicara a observar y aprender sobre la tuberculosis, también lo hizo con el sistema de aguas y de su purificación por lo que se puso en contacto con el ingeniero que lo había logrado en Liberty con el fin de instalarlo en San José; con ese fin, empezó a escribir a La Prensa Libre, para que fueran preparando el terreno para las obras se podrían llevar a cabo, tiempo después desde su puesto en la Municipalidad de San José pudo realizar este proyecto..22

Otro aporte importante del doctor Durán se palpa en la biblioteca que poseía el Sanatorio Loomis, donde debe haber habido excelente literatura sobre la tuberculosis. También es probable que en la larga temporada que el doctor pasó en Liberty observara todo lo referente a la administración de un sanatorio, conocimientos que luego le sirvieron para la fundación del sanatorio en Tierra Blanca. Aspecto que se refuerza al observar las siguientes fotografías, en las que se aprecian las semejanzas de ambos sanatorios el de Liberty y el de Tierra Blanca.

21 La Gaceta Nº114, año XXV, p487 del 20 de mayo de 1903.

22 Carlos Pupo. El Doctor Carlos Durán.( San José, Imprenta Nacional ,1924), 18. 


\section{IX) concasoso

Indexaciones: Repositorio de Revistas UCR, DIALNET, Latindex, REDALYC Directorio y recolector de recursos digitales del Ministerio de Cultura de España, Directory of Open Access Journals.

Diálogos Revista Electrónica de Historia ISSN 1409-469X. Número especial 2008. Dirección web: http://historia.fcs.ucr.ac.cr/dialogos.htm

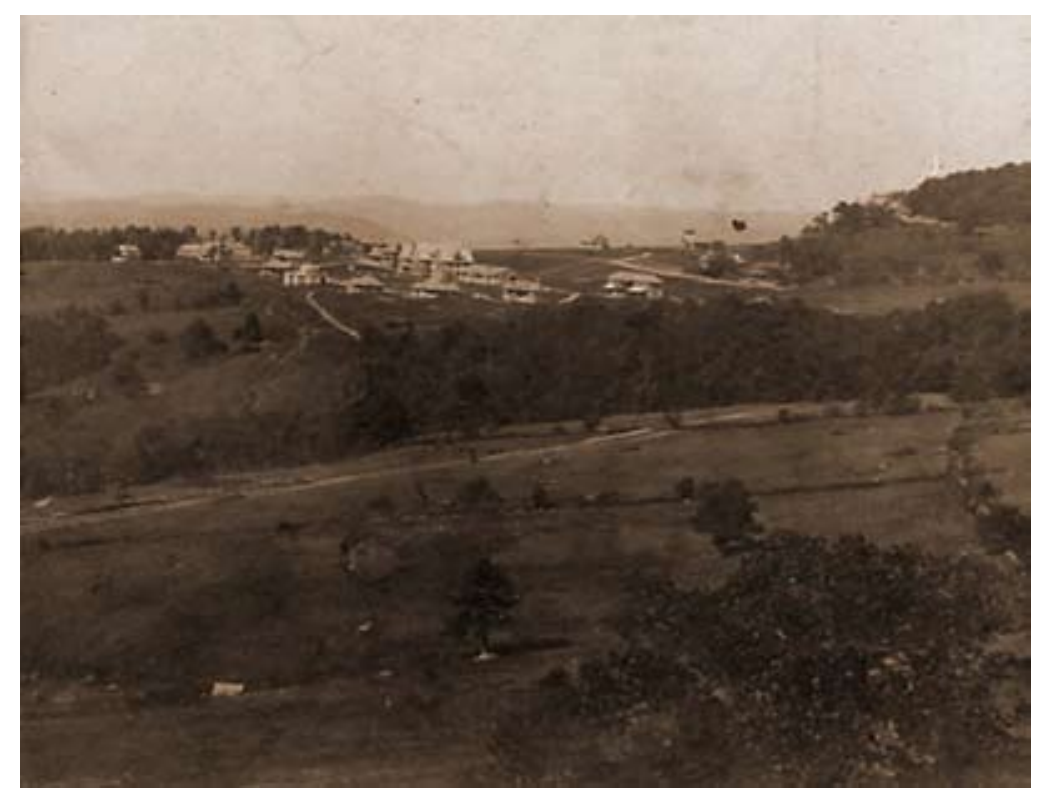

Lamina $N^{\circ} 1$ del Sanatorio de Loomis en Liberty New York, 1903

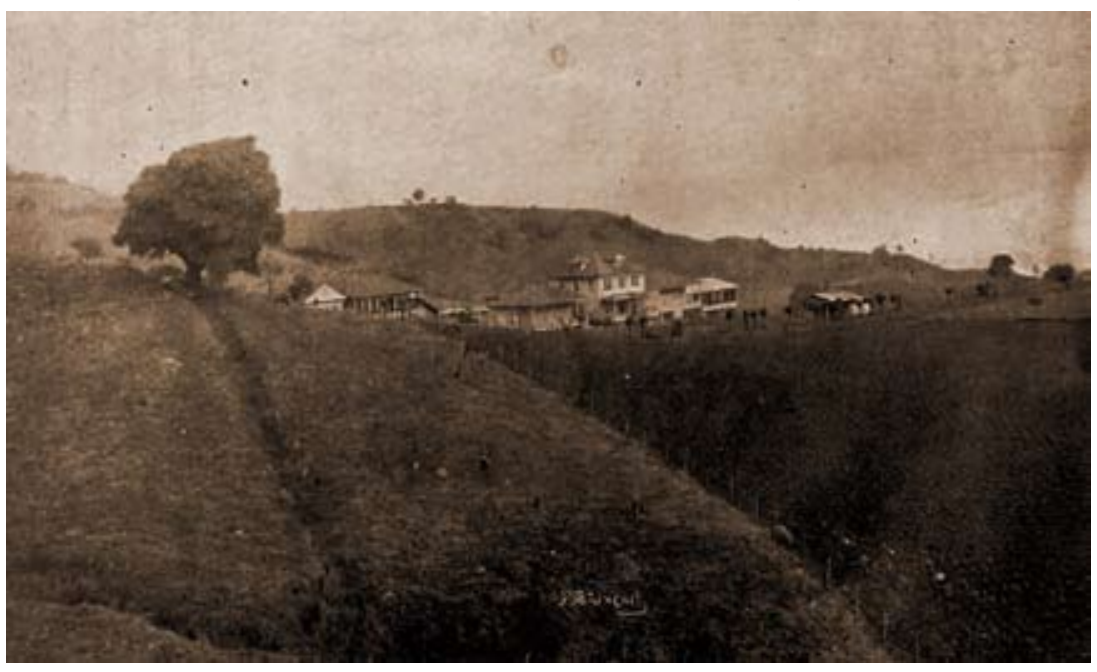

Lámina N² del Sanatorio Carit en Tierra Blanca de Cartago 1924

En 1912, Durán regresó al Congreso de la República y preparó un proyecto para la creación de un sanatorio con la ayuda del licenciado Manuel Coto Fernández. Así, el 16 de agosto de 1915 se aprobó la creación de un sanatorio. En relación con esto el doctor argumentó lo siguiente: 
En Costa Rica la mayoría de las personas se hallan afectadas por la tuberculosis, y las estadísticas demuestran que este mal ocupa uno de los primeros lugares entre las causas de defunción...La enfermedad puede curarse, en sus comienzos con una serie de medidas como cambio de clima, reposo, y alimentación adecuada. Esto no lo pueden hacer las personas de escasos recursos, porque su posición pecuniaria los priva de esos elementos."23

El doctor Durán agregó que el sanatorio podría cumplir con varios fines como aislar muchos focos de infección, servir de escuela de educación antituberculosa, permitir la curación de individuos que sin él estarían condenados a muerte y, además, libraría a las instituciones de caridad del cuidado y de los gastos ocasionados por enfermos a los que ningún alivio podía ofrecer, debido a las condiciones inadecuadas de sus instalaciones. ${ }^{24}$

Al aprobar el proyecto para la creación de un sanatorio para los enfermos de tuberculosis, el Congreso en el artículo 1 de la ley señaló: “Créase con carácter de Nacional un Sanatorio de Tuberculosos destinado a albergar, aislar y procurar la curación de los tuberculosos de la República. El establecimiento se llamará SANATORIO CARIT”. ${ }^{25}$ La ley indicó también que la adquisición del terreno, construcción del edificio, así como la dirección y administración, y equipo del sanatorio estaría a cargo de una junta compuesta por cinco miembros, tres de los cuales debían ser médicos, otro abogado y el otro ingeniero. La junta se llamaría “Junta del Sanatorio.” Los nombramientos lo realizaría la Facultad de Medicina, serían en carácter gratuito y solo el presidente tendría sueldo. Esta junta debía establecer un reglamento para el funcionamiento del sanatorio. $^{26}$

Para que el nuevo centro de salud se pudiera mantener, el Congreso estableció un impuesto de un "uno por ciento sobre las utilidades netas que queden anualmente a las instituciones bancarias o sucursales de bancos extranjeros, establecidos en el país y a las casas de préstamo. Este impuesto regirá desde el primero de enero de 1916.”27 Además, se puso un impuesto de cinco céntimos de colón por cada litro de cerveza que se fabricara en el país para apoyar al sanatorio.

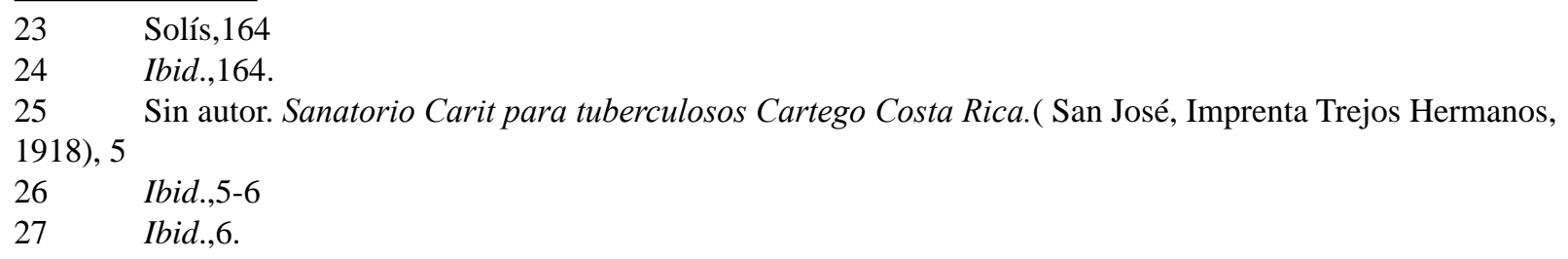


De igual manera, la institución podría recibir donaciones y los enfermos que lo pudieran hacer pagarían una pensión.

Otro ingreso posible fue cubierto por la ley que en el artículo 9 señala: "El establecimiento será el heredero obligado y universal de todos los tuberculosos que mueran en el Sanatorio, abintestato y sin parientes sucesores." ${ }^{28}$

La construcción se financió por medio de un empréstito de ф 200.000 que afectó en garantía las rentas que se que se le adjudicaron. Con respecto a la junta del sanatorio, esta se nombró en la sesión ordinaria de la Junta General de la Facultad de Medicina del 8 de setiembre de 1915 y ahí por aclamación fue nombrado como presidente el doctor Carlos Durán Cartín y para completar la junta los doctores Luis Paulino Jiménez y Teodoro Picado junto con el ingeniero Lucas Fernández y el licenciado Claudio González Rucavado. ${ }^{29}$

Una vez establecida, la junta se dedicó a buscar el lugar idóneo y los medios necesarios para construir el sanatorio. Fue necesario obtener un empréstito en el Banco Anglo Costarricense por ciento cincuenta mil colones, al principio al 10\%, pero a partir de enero de 1918 al 12\% anual. Por otra parte, el doctor Durán obtuvo un préstamo de cincuenta mil colones con doña Elena viuda de López Calleja al 9\% anual y ya se habían recogido de los impuestos, que por ley le tocaban a la institución, $\notin 78.631,84$. Esta suma al tipo de cambio legal de Costa Rica sobre oro americano era en ese momento el de $\phi 2,15 .^{30}$

En cuanto al lugar escogido para el sanatorio, la junta revisó varias opciones contemplando siempre las observaciones de Durán como la altura de los montes, los vientos imperantes, la humedad del aire, la temperatura, las horas de sol y otros datos importantes que se debían tomar en cuenta para el establecimiento del sanatorio. ${ }^{31}$ Luego de hacer varias incursiones por las montañas que rodean a San José se escogió el lugar llamado “Los Horcones” en Tierra Blanca de Cartago que, además de considerarlo idóneo, la Municipalidad de Cartago ofreció ayuda con el terreno y contribuir con el arreglo del camino.

El lugar tenía condiciones climatológicas buenas de acuerdo con los estudio realizados por Elías Leiva: agua potable, porque el lugar está protegido por una colina que impedía los vientos del norte; la exposición del terreno al lado sur era conveniente para obtener mayor

\begin{tabular}{ll}
\hline 28 & Ibid., 7. \\
29 & Ibid., 9-10. \\
30 & Ibid.,17. \\
31 & Ibid.,17-18.
\end{tabular}


número de horas sol; era el sitio más cercano al ferrocarril al Atlántico, también su altura era la media para poder crear un sanatorio, porque el terreno era arenoso y, por lo tanto, bueno para crear drenajes, porque el aire seco y la temperatura eran los indicados para la curación de la tuberculosis y porque la experiencia había demostrado que una gran proporción de enfermos que habían ido a Tierra Blanca buscando la cura de la tuberculosis lo habían logrado. ${ }^{32}$

Aprovechando el último viaje del doctor Durán a Estados Unidos y la visita que realizó a diferentes sanatorios de tuberculosos de los que trajo los planos del Ewdowood Sanatorium Towson, que inspiraron los planos para el Sanatorio Carit. El ingeniero Lucas Fernández hizo los estudios y los cálculos, el dibujante fue Guillermo Gargollo y como constructor fungió Francisco Zúñiga.

En cuanto a la construcción de los edificios, se utilizaron maderas de nuestro país como roble negro, cedro y pochote, así como la caña de Castilla; también con madera nacional se fabricaron armarios, sillas, mesas, estantes para uso de los médicos. La ropería se trajo de los Estados Unidos de Norte América, así como la loza, cristalería, clavos para techumbre, tubería, cerraduras, bisagras, lavabos, calefactores, excusados fijos y portátiles, aparatos higiénicos para recoger la ropa sucia, mesitas de noche, pintura, camas de hierro para enfermos, perlina, escobas, aparato de succión de polvo, depósitos portátiles para basura, hilo, agujas y botones, imperdibles y toallas. Y en Costa Rica se hicieron las sábanas, almohadas, batas y colchones. ${ }^{33}$ Los productos que fueron pedidos a New York, en su mayoría fueron a través de la casa de Montealegre y Bonilla en esa ciudad y traídos desde Limón por medio del ferrocarril de la Northern Railway Company que concedió una rebaja en las tarifas de un cincuenta por ciento, en todo lo necesario para el funcionamiento del sanatorio.

Para 1918 ya estaban construidos tres edificios principales y un galerón cerrado, que incluían la administración y los pabellones de hombres y mujeres, cuya ampliación futura se dejó planteada. En el caso de la cocina, esta fue construida de bahareque de mezcla y de caña de Castilla. "Esta clase de materiales los aconsejó la prudencia, pues ya se sabe cuanto tiembla en Costa Rica.” ${ }^{34}$ En el caso de la administración quedó espacio debajo para poder construir no solo bodegas sino hasta habitaciones para algunos usos.

\begin{tabular}{ll}
\hline 32 & Ibid., 25-26. \\
33 & Ibid., 35. \\
34 & Ibid.
\end{tabular}




\section{IIX) concenso \\ of CENTROAMERKCANO \\ LL IIISIORIA}

Indexaciones: Repositorio de Revistas UCR, DIALNET, Latindex, REDALYC Directorio y recolector de recursos digitales del Ministerio de Cultura de España, Directory of Open Access Journals.

Diálogos Revista Electrónica de Historia ISSN 1409- 469X. Número especial 2008. Dirección web: http://historia.fcs.ucr.ac.cr/dialogos.htm

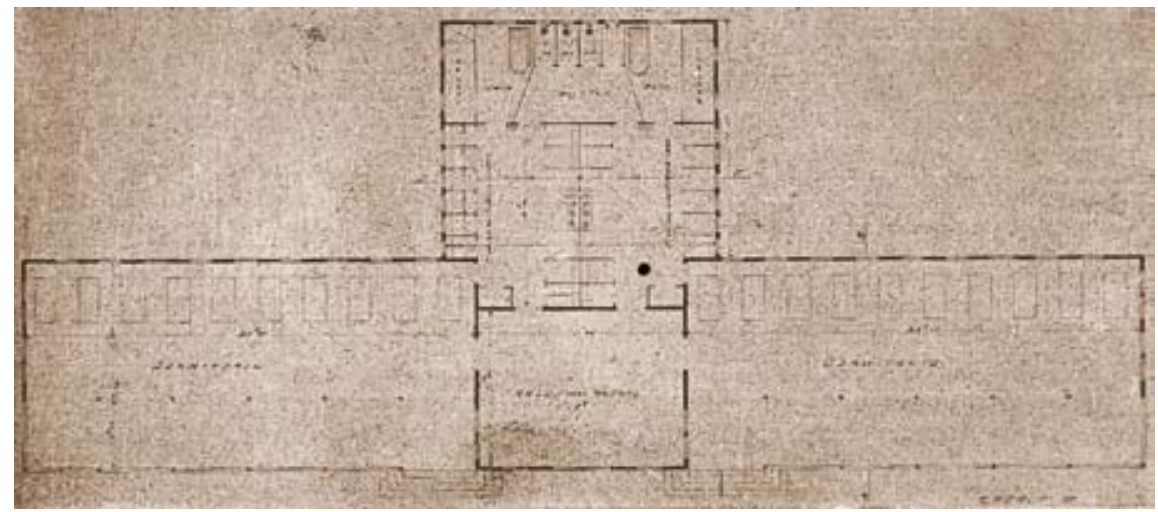

Lámina $\mathrm{N}^{\circ} 3$ Plano de pabellón de enfermos

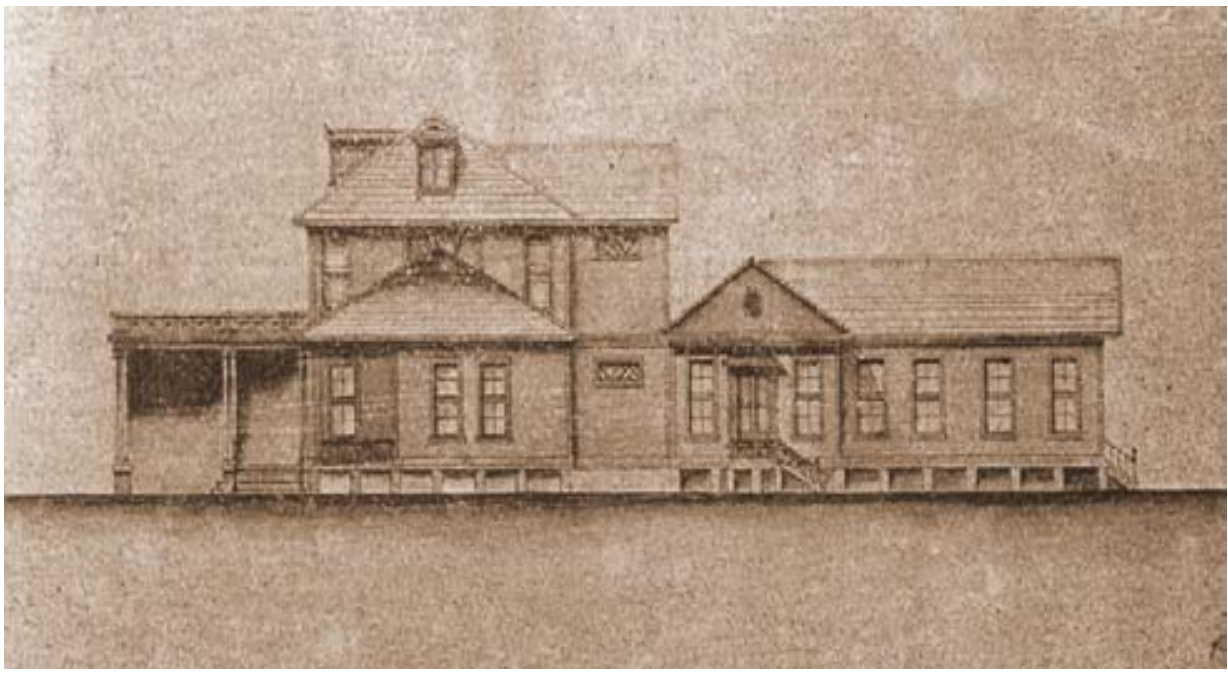

Lámina N4 Edificio de Administración- fachada lateral, 1924 


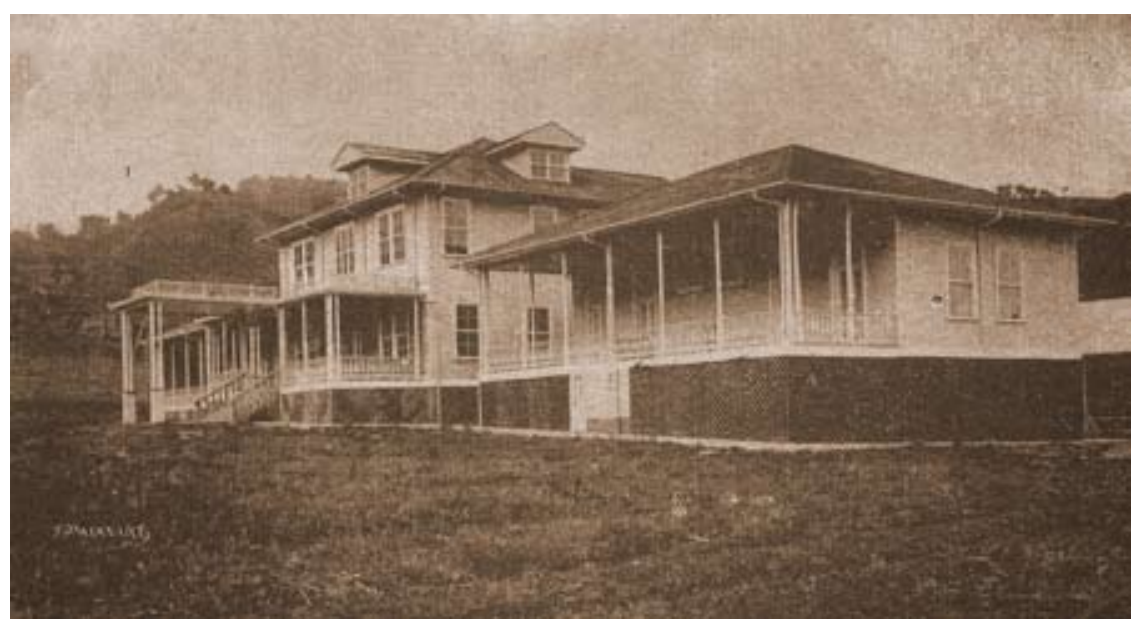

Lámina N5 Vista del frente de la administración , 1924

Como se puede observar en la lámina No. 5 el espacio entre el suelo y la primera planta era grande y efectivamente con el transcurso de los años se dedicó a varios usos entre ellos el de bodega como se fue planeado, pero por los vestigios que hoy existen parece que hasta algunas oficinas estuvieron ahí. Los boquetes de las puertas son bajos, pero una vez que ya uno ingresa la persona de una altura media puede moverse sin necesidad de agacharse.

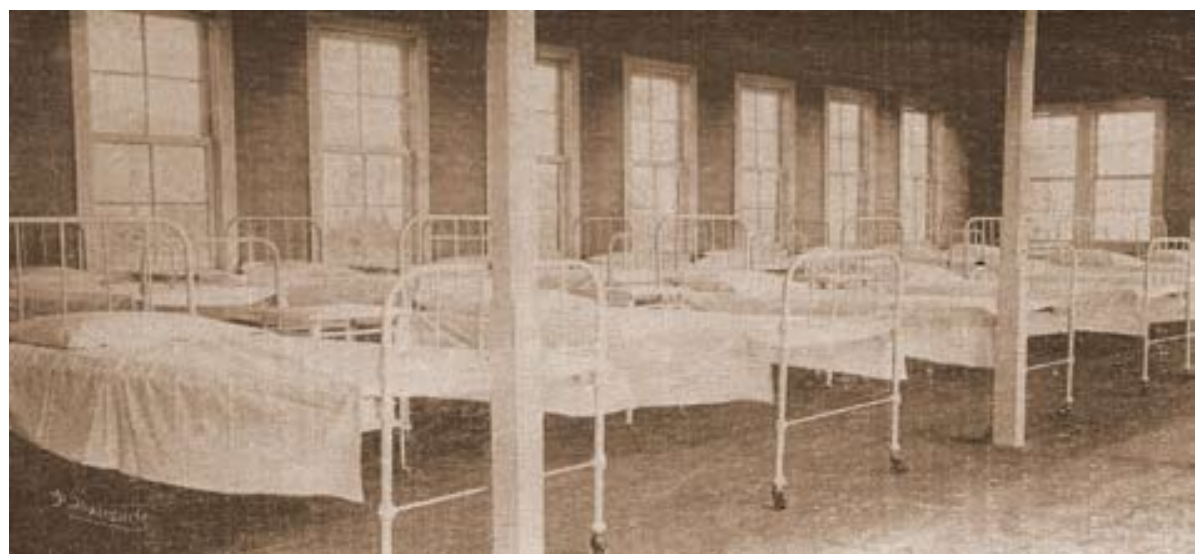

Lámina $\mathrm{N}^{\circ} 6$ Dormitorio de enfermos, 1924

En el edificio de la administración a la izquierda (Lámina No.5) se encontraba la sala de recibo, un zaguán que conducía a un salón de recreo; también estaban la biblioteca, en la parte de atrás, la cocina, el comedor de sirvientes y los cuartos de la loza y dos despensas. A la derecha de la entrada, se encontraba el consultorio médico con su cuarto de exámenes, el 
comedor del médico, independiente del consultorio. Había un pasadizo hacia el ala derecha, donde permanecían los pacientes en observación, tanto hombres como mujeres, pero separados por dos cuartos para guardar ropa. En el segundo piso, había seis habitaciones, dos roperías, dos baños y dos inodoros. Ahí se encontraban también dos baños y dos inodoros y los dormitorios del personal superior del sanatorio. En la bohardilla había cuatro miradores y se destinaba a los dormitorios de los sirvientes. ${ }^{35}$

\section{Organización general del sanatorio a nivel interno y externo}

Como se ha indicado, a la cabeza de la organización del sanatorio estuvo una junta directiva compuesta por cinco miembros. De ellos tres eran médicos, uno abogado y el otro ingeniero. Como presidente de esta junta estuvo hasta su muerte en 1924, el doctor Durán, quien a pesar de ser el único miembro de la directiva con salario, lo donó siempre al mismo sanatorio.

En cuanto a la organización general del servicio, existió un médico nombrado por la junta del sanatorio que despachaba en San José, para atender ahí la consulta de los pacientes que querían ingresar. Ese mismo médico debía desplazarse a Cartago un día por semana con los pacientes por internar. La idea era recibir pacientes declarados tuberculosos, pero con posibilidades de curación y cuidar por su trasladado al sanatorio. En cuanto al superintendente del sanatorio Carit, este también era nombrado por la junta, su función principal consistía en administrar el sanatorio y ejecutar las órdenes que le diera la junta. Debía disponer convenientemente de los edificios, jardines, huertos o campos y muebles del sanatorio, y presentar un presupuesto cada seis meses y sujetarse a él. Dentro de sus funciones también estaban las siguientes:

“...llevar una historia clínica de cada enfermo con anotaciones semanales acerca del peso, del estado de los pulmones y de la laringe y demás órganos que están afectados, todo lo más completo posible; llevar y conservar en el mayor orden en el archivo del Sanatorio, las historias clínicas, los datos de la administración, informes, observaciones personales, tratamientos y cuantos datos considere de utilidad." 36

Además según señala la misma fuente se procuraba que al ingresar el enfermo al sanatorio fuera acogido con palabras de esperanza y que se le explicara todo lo concerniente al 35 Ibip.,40-41.

36 Ibid.,51. 
funcionamiento de la institución. En cuanto a la disciplina de los enfermos y del personal, debían guardar un orden severo para el buen funcionamiento del sanatorio.

Por otra parte, el tratamiento constaba de un régimen dietético-higiénico; así fue este desde su fundación e incluso hasta en 1924. Se limitaba "a combatir ciertos síntomas penosos o complicaciones, y en algunos casos solamente a ensayar de hacer más efectiva la resistencia del organismo. Con este último fin se usaron más especialmente los recalcificantes, el yodo y el arsénico.”37

Tres años después en una entrevista, el doctor Jorge Sáenz, quien había sido superintendente del sanatorio desde su fundación, señaló que se continuaba aplicando el mismo tratamiento,

“...que es el mismo que han adoptado hoy todos los sanatorios importantes de Estados Unidos y de Europa está basado en el régimen dietético-higiénico y consiste esencialmente en reposo, aereación continua y buena alimentación. Es un tratamiento indirecto puesto que viene a dominar la infección fortaleciendo el organismo.”38

Este tratamiento según indicó Sáenz, si no cura en todos los casos, es el que menos decepciones da al médico y a los pacientes. El problema era que los pacientes llegaban generalmente muy tarde al sanatorio, porque se engañaban ellos mismos, conciente o inconcientemente, o los engañaban los charlatanes ofreciéndoles curarlos. También existía el miedo a la decisión de dejar a su familia e irse a un lugar alejado y ser sometido a una disciplina severa de vida. Para Sáenz si muchos pacientes hubieran llegado más temprano al sanatorio, su cura podría haber sido más efectiva.

En relación con las estadísticas de los sanatorios del mundo, Sáenz señaló: “Comparando imparcialmente los resultados obtenidos del Sanatorio Carit con los consiguientes establecimientos similares en diferentes países de Europa y los Estados Unido, se ve que aquellos no son en manera alguna inferiores.”’39 También dijo que en las estadísticas se debían contemplar las condiciones de admisión de los pacientes de acuerdo con el estado de avance de la enfermedad con el fin de tener un conocimiento más confiable de las estadísticas.

37 Jorge Sáenz. Informe del superintendente .(San José Costa Rica, Imprenta y Librería Trejos Hnos, 1924),8.

38 Jesús Mata Gamboa. El sanatorio Carit de Tierra Blanca, la gran Institución de Beneficencia Nacional. En El Maestro. Tomo 1, Nº II.(San José, Imprenta María v. de Lines, 1927),337.

39 Ibid ., 342. 
Al sanatorio llegaban pacientes de todas las provincias de Costa Rica, así, en 1924 ingresaron 39 de San José, 16 de Cartago, 5 de Alajuela, 2 de Heredia, 4 de Puntarenas, 3 de Limón y 9 de Guanacaste. Además, en el informe de ese año se reportó el ingreso de 2 colombianos, 1 cubano, 1 chino, 2 españoles, 1 inglés, 2 panameños, 1 persa, 5 nicaragüenses y 2 salvadoreños. ${ }^{40}$ Lo que muestra que este centro daba servicio a todo Costa Rica y también a extranjeros que como en el caso de los centroamericanos carecían de un centro semejante.

El sanatorio continuó llamándose Carit hasta 1931 cuando se le cambió el nombre a Sanatorio Durán en homenaje al gran impulsor de la obra para la cura de la tuberculosis.

\section{La vida cotidiana en el sanatorio}

Los enfermos debían guardar un gran reposo como parte de su curación, pero cuando estaban mejor, se les recomendaba que hicieran pequeñas caminatas para fortalecerse, no sin dejar de señalar que el terreno tan quebrado del sanatorio no permitía que fueran muy largas. También eran llevados a recibir el sol a los jardines para lograr los beneficios de la radiación solar.

En cuanto a las distracciones era posible ver películas suministradas por la empresa Urbini y Facio, que por medio de su agente en Cartago, Ovidio Loría, en varias ocasiones las facilitaban sin cobrar alquiler alguno. También los pacientes tenían acceso a revistas y libros que se recibían en el sanatorio como donaciones.

Algunos pacientes podían realizar trabajos poco pesados en la huerta y en el jardín cuando su estado lo permitía sin correr peligro. Esta práctica no fue fácil porque los enfermos con tanto reposo perdían el hábito del trabajo. Entonces, el doctor Sáenz propuso que se contratara un maestro de trabajos manuales para que los pacientes sin utilizar mucha fuerza, eso sí era necesario facilitarles los materiales, ya que la mayoría era muy pobre y no podían adquirirlos por cuenta propia. De esa manera, se procuró que produjeran algunos objetos que pudieran vender (previa esterilización), lo que podría ser para algunos un medio de procurarse algunos recursos para ellos o sus familias y, por otra parte, una manera eficaz de combatir el fastidio y la depresión moral provenientes de la falta de ocupación que tan perniciosos efectos ejerce sobre algunos. ${ }^{41}$

Otro de los momentos de reunión de los enfermos era a la hora de recibir sus alimentos; el sanatorio estaba de manera que hubiera comedores de acuerdo con el sexo y la edad, es decir, $40 \quad$ Sáenz., 7

41 Sáenz op cit p.9 
de hombres adultos, de mujeres adultas, de niños y de jóvenes por separado.

Es probable que la vida en el sanatorio transcurriera dentro una cierta calma por el reposo que requerían los enfermos, de la esmerada alimentación y de los largos periodos la aire libre para poder disfrutar de los beneficios del clima, de los vientos y del sol como parte importante de su curación.

\section{Las ampliaciones de sanatorio Durán}

Con el paso del tiempo el sanatorio se fue ampliando y ya sus materiales empleados en las nuevas construcciones fueron diferentes, su carácter victoriano debió dar paso al ladrillo y al cemento que hicieron su ingreso como materiales novedosos.

Hasta 1973 hubo ampliaciones en el sanatorio. Actualmente, lo que queda son vestigios de los que fue uno de los centros más importantes de Centroamérica para curar la tuberculosis. Sus edificios están en ruinas, el descuido y la falta de respeto por un pasado, no solo del sector de la salud de nuestro país, sino de todos los costarricenses que lo han permitido.

El sanatorio fue entregado a UPA Nacional, parece que siembran algo. Hasta los árboles de eucalipto, que se habían sembrado para que ayudaran con su olor a curar a los enfermos, fueron cortados; los pinos también porque podían hacer daño a un muro; sin embargo, no se consideró que el edificio necesitaba reparaciones y que los árboles eran necesarios para dar un ambiente de frescura y tranquilidad.

Algunas fotos muestran como se levantaron los nuevos pabellones, algunos de ellos por medio de maratónicas nacionales; de ellos quedan tan solo ruinas. 


\section{OTO $9^{\circ}$ CONGRESO
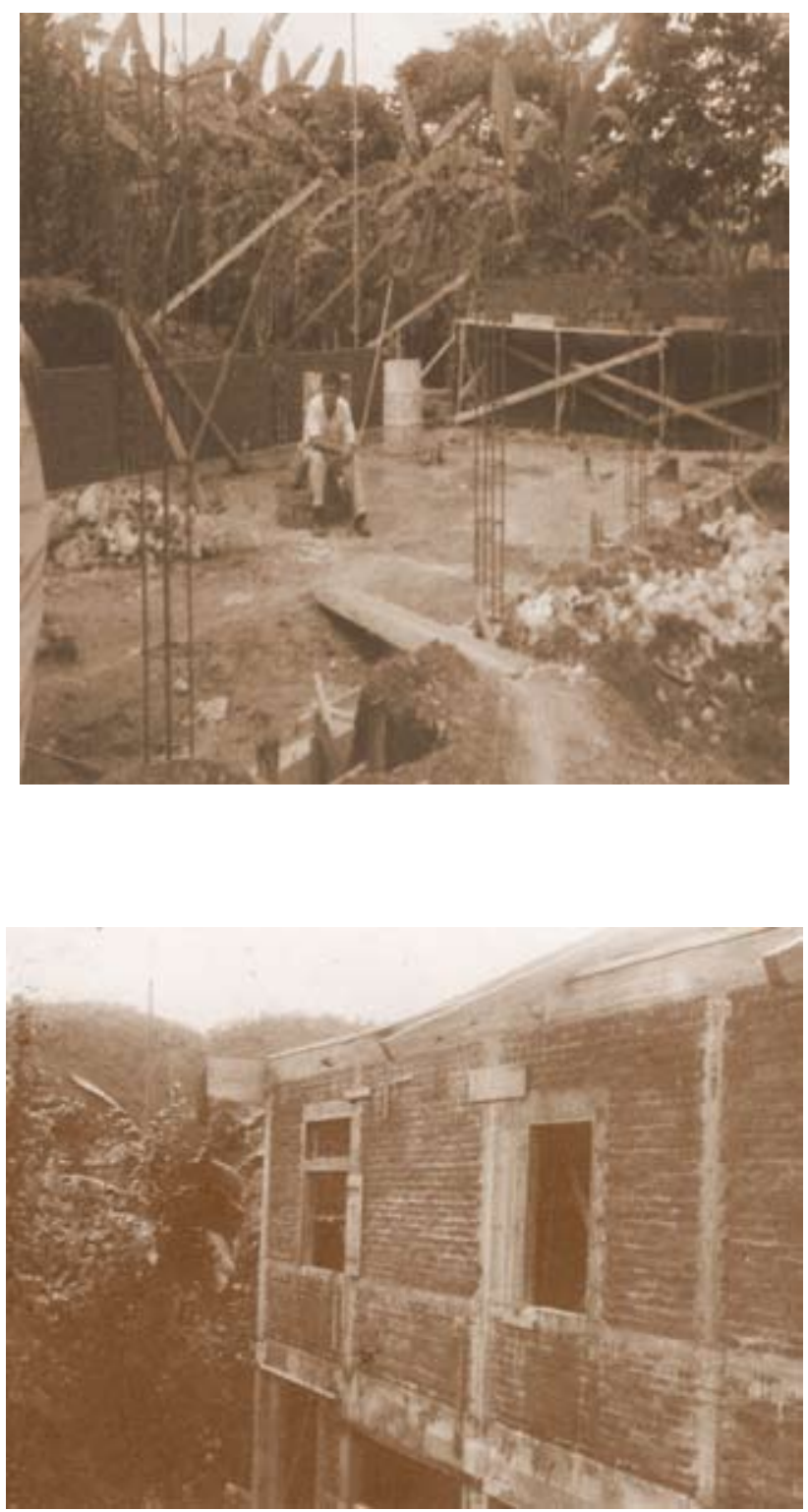

Láminas $N^{\circ} 7$ y 8 de ampliaciones del sanatorio

El Sanatorio Loomis también fue cerrado y sus edificios no se han utilizado para otro fin. Aparentemente, por fuera las edificaciones no se muestran tan deterioradas como las del Sanatorio Durán, debido probablemente a los materiales de construcción, pero también es una lástima que estén tan abandonados ambos centros sanitarios a pesar de haber sido tan importantes 


\section{9) $9^{\circ}$ CONG R E SO \\ of $/ \mathrm{S}$ L IIISIOIR IA}

Indexaciones: Repositorio de Revistas UCR, DIALNET, Latindex, REDALYC Directorio y recolector de recursos digitales del Ministerio de Cultura de España, Directory of Open Access Journals.

Diálogos Revista Electrónica de Historia ISSN 1409-469X. Número especial 2008. Dirección web: http://historia.fcs.ucr.ac.cr/dialogos.htm

en los momentos en que el avance de la tuberculosis los hizo indispensables.

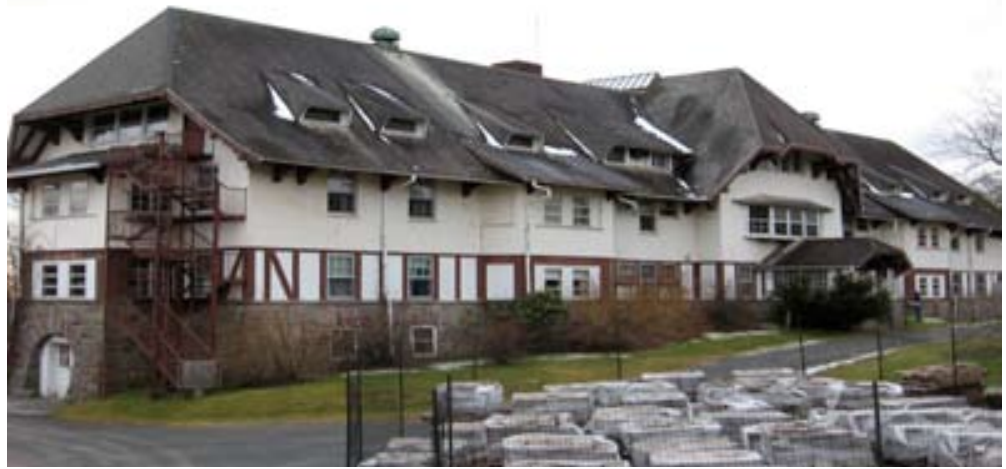

Lámina N9 Sanatorio Loomins en 2007

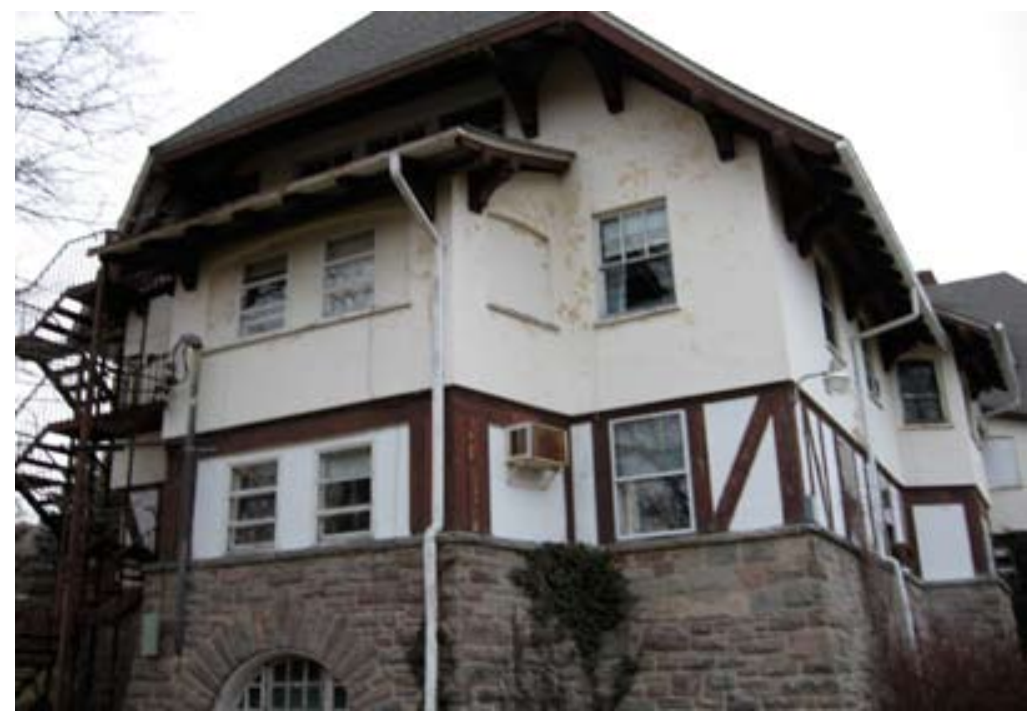

Lámina Nº10 Sanatorio Loomis en 2007 

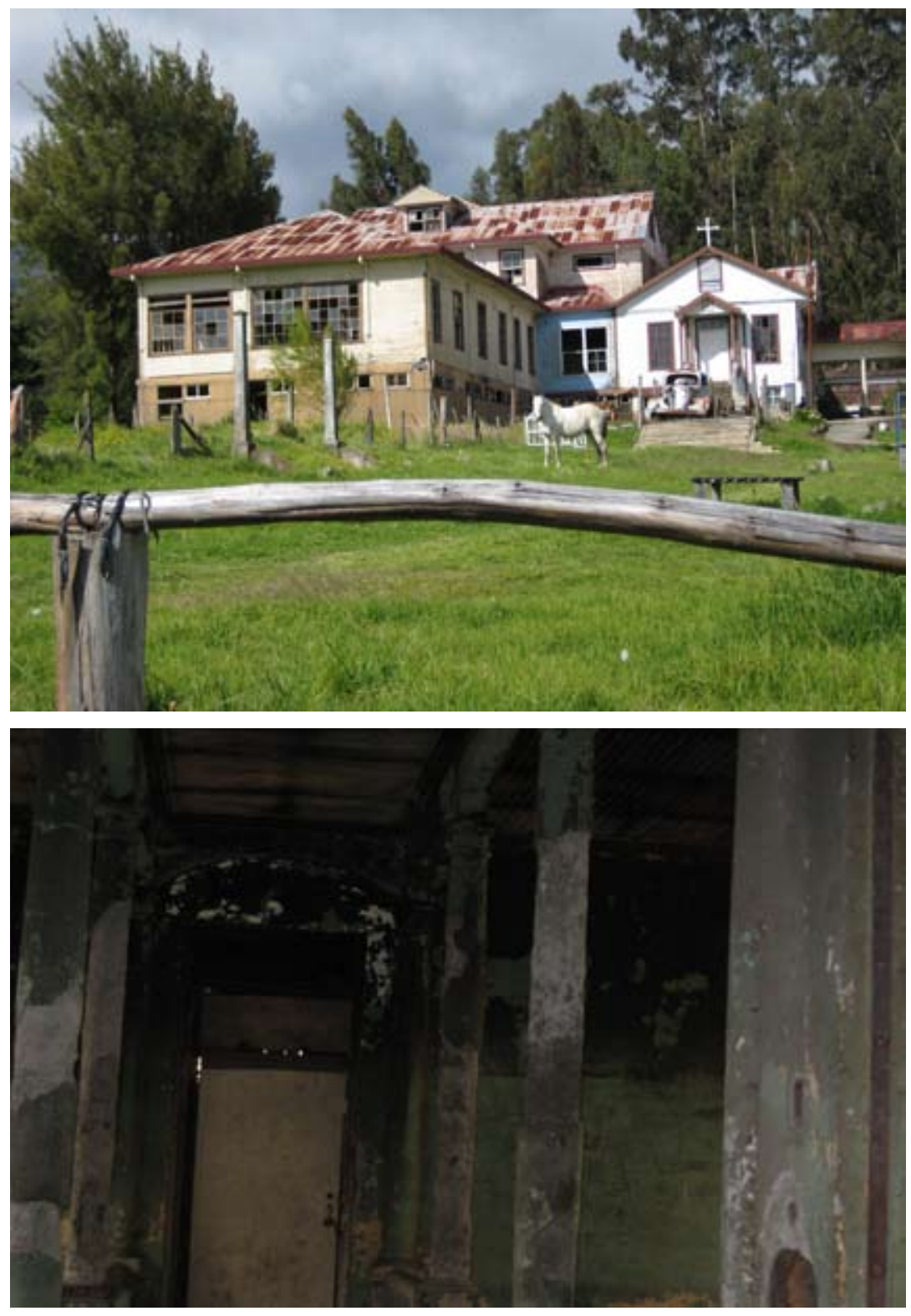

Láminas N¹1 y 12 Sanatorio Durán en 2008

Otra pregunta que surgió con este trabajo fue qué ha pasado con la tuberculosis y cuál es el estado de esa enfermedad en Costa Rica. El lunes 24 de marzo, el día mundial de la tuberculosis, en una pequeña columna del periódico la Nación, señaló que la tuberculosis ha tomado nuevamente fuerza. En América es la causante anual de la enfermedad de 331.000 persona y de ellas mueren más de 40.000. A nivel mundial, la cifra ronda los 9 millones de casos 
con tuberculosis y los 2 millones de muertos. Para Costa Rica señala la misma fuente que el Ministerio de Salud anunció un plan para disminuir de 12 casos a menos de 5 por 100.000 y, finalmente, eliminar la enfermedad en el 2.015. Sin embargo, aún no se ha sabido nada de los resultados que se han obtenido recientemente. ${ }^{42}$

\section{Conclusiones}

Con este trabajo se ha podido constatar la repercusión que tuvo la tuberculosis a nivel mundial y como a través del tiempo se ha buscado su cura, desde los reyes taumaturgos hasta la creación de los sanatorios, donde con buena alimentación y con el beneficio de los aires de la montaña, junto con el reposo se lograba que los pacientes con la enfermedad en estado no muy crítico pudieran recuperar la salud. Casos como el del doctor Loomis que logró curarse y el de la hija del doctor Durán, Elena, quien sobrevivió a la enfermedad por 42 años ya que murió en 1945 y de otra enfermedad.

Sobre el Sanatorio Durán y su aporte a la lucha contra la tuberculosis, se considera que este centro de salud es un buen ejemplo de la preocupación tanto de los médicos, del gobierno y de la ciudadanía costarricenses por prevenir, mejorar y obtener la curación de los enfermos. Los médicos no solo eran conocedores de la medicina, sino que buscaron los medios para crear centros hospitalarios para curar y capacitar también a enfermeras y doctores en los nuevos avances de la medicina, lo que convirtió a Costa Rica en un país con una medicina que hoy llamaríamos de punta.

Ha sido también importante comprobar que nuestros galenos no se quedaron con los estudios necesarios para graduarse como profesionales en el campo de la medicina, sino que siguieron preparándose y viajando a Europa y Estados Unidos en búsqueda de conocimientos nuevos para mejorar la salud de sus pacientes.

En cuanto a la construcción del sanatorio, es interesante comprobar como se procuró cumplir con todos las condiciones requeridas para establecer un centro de acuerdo con los conocimientos de aquel momento: desde escoger las mejores maderas para que soportaran la humedad de la zona, como el pochote, hasta todos los catres y los utensilios para el cuidado de los enfermos, que fueron traídos desde New York por medio de la firma establecida en esa ciudad de Montealegre y Bonilla. También es destacable la búsqueda del lugar para construir

$42 \quad$ Yalena de la Cruz La Nación, Lunes 24 de marzo del 2008, 47. 
el sanatorio por las montañas que rodean el Valle Central; lo mismo que los análisis sobre la potabilidad del agua y la decisión de escoger un sitio lejos de donde se abastecían las ciudades importantes de agua para no contaminar esas fuentes y así no trasmitir la enfermedad.

Por eso, una vez conocido todo ese esfuerzo que realizaron nuestros antepasados por crear un sanatorio comparable con los mejores centros de cura para la tuberculosis, duele comprobar que no se hayan respetado ni cuidado sus instalaciones a pesar de encontrarse en buenas condiciones cuando se cerró y actualmente permanecen en el desamparo y en el olvido. Por eso, es normal preguntarse, si un lugar que fue orgullo nacional y ha sido relegado al olvido, ¿qué se puede esperar en nuestro país sobre la posibilidad de conservar lo que no se ha destruido?

Ante esa interrogante propongo restaurar una parte de las instalaciones del Sanatorio Durán para convertirlo en un centro de salud o de enseñanza, pero que conserve su dignidad como sanatorio y que se permita mostrar el respeto y el agradecimiento merecido hacia quienes lo construyeron con cariño y esfuerzo, especialmente se debe resaltar a su principal promotor el doctor Carlos Durán Cartín. 